\title{
Design and Application of Intelligent Cloud Dis- ease-Diagnostic System Based on Non-Coding Amino Acid Mass Spectrometry
}

\author{
Xingyuan Wang*, Yuqiao Zhang and Ming Mingming \\ School of Management, Shandong University, China \\ *Corresponding author: Xingyuan Wang, School of Management, Shandong University, China
}

\begin{tabular}{|c|c|}
\hline ARTICLE INFO & ABSTRACT \\
\hline Received: 幽 December 13, 2019 & Citation: Xingyuan W, Yuqiao Zhang, Ming M. Design and Application of Intelligent \\
\hline Published: 幽January 06, 2020 & $\begin{array}{l}\text { Cloud Disease-Diagnostic System Based on Non-Coding Amino Acid Mass Spectrometry. } \\
\text { Biomed J Sci \& Tech Res 24(1)-2020. BJSTR. MS.ID.004001. }\end{array}$ \\
\hline
\end{tabular}

\section{Short Communication}

With the society's increasingly urgent need for precision medical services [1,2], only relying on a wealth of practical experience and higher level of knowledge of medical experts and professors is limited, while the traditional diagnosis and treatment technology is limited [3]. At present, imaging, biochemical and other methods in the diagnosis of diseases have large errors. The non-coding amino acid markers were identified and measured by mass spectrometry. The non-coding amino acid markers of a large number of tumor patients were integrated with the immune system diversity index, and the cancer tissue diagnostic markers based on the protein change index were carved out, so as to establish the diagnostic criteria based on the non-coding amino acid markers and immune system diversity index [4,5]. In this study, a cloud diagnosis medical information system is constructed based on the non-coding amino acid mass spectrometry database, the internal structure and application process of the cloud diagnosis platform are designed, and the disease intelligent cloud diagnosis system and information service mechanism based on the non-coding amino acid mass spectrometry analysis are constructed.

Intelligent Diagnostic Cloud Information System Architecture

The disease cloud diagnosis information system based on non-coding amino acid mass spectrometry is an application information service system built based on a unified data center. Its data platform mainly has three functions: data collection, data processing, and data management. The data were obtained from the non-coding amino acid mass spectrometry Research Institute and the cooperation hospital. Research institutions mainly provide non-coding amino acid mass spectrometry standardized data as the basis for diagnosis; hospitals provide basic information, patient related information, and clinical information. The collected data need to be transmitted, processed, analyzed, transformed and analyzed intelligently based on the deep convolution neural network method [6]. Data management mainly includes hospital information management, patient data management, cost management, contact information management and non-coding amino acid mass spectrometry data dictionary management services. The whole information system is built under the support of infrastructure, which mainly includes cloud storage server, cloud computing server, communication network, etc. The whole system architecture is as shown in the Figure 1.

Cancer Screening Technology Path Based on Mass Spectrogram Identification of Deep Convolution Neural Network Method

Mass spectrogram contains a lot of information [7]. If we use computer image processing technology to process it, it is still at the level of analysis and processing. In the era of rapid development of artificial intelligence, using deep learning technology, using convolutional neural network framework [8] to iterate and abstract the mass spectrum, iterating the original signal from lowlevel abstraction to high-level abstraction, the higher the level of abstraction, the more conducive to the appearance of high-level 
representation, and the higher the accuracy and reliability for medical detection [9]. Based on the research of deep convolution neural network framework and non-coding amino acid detection mass spectrometry technology, we propose a mass spectrum convolution neural network recognition and diagnosis technology. The technical path is shown in Figure 2.

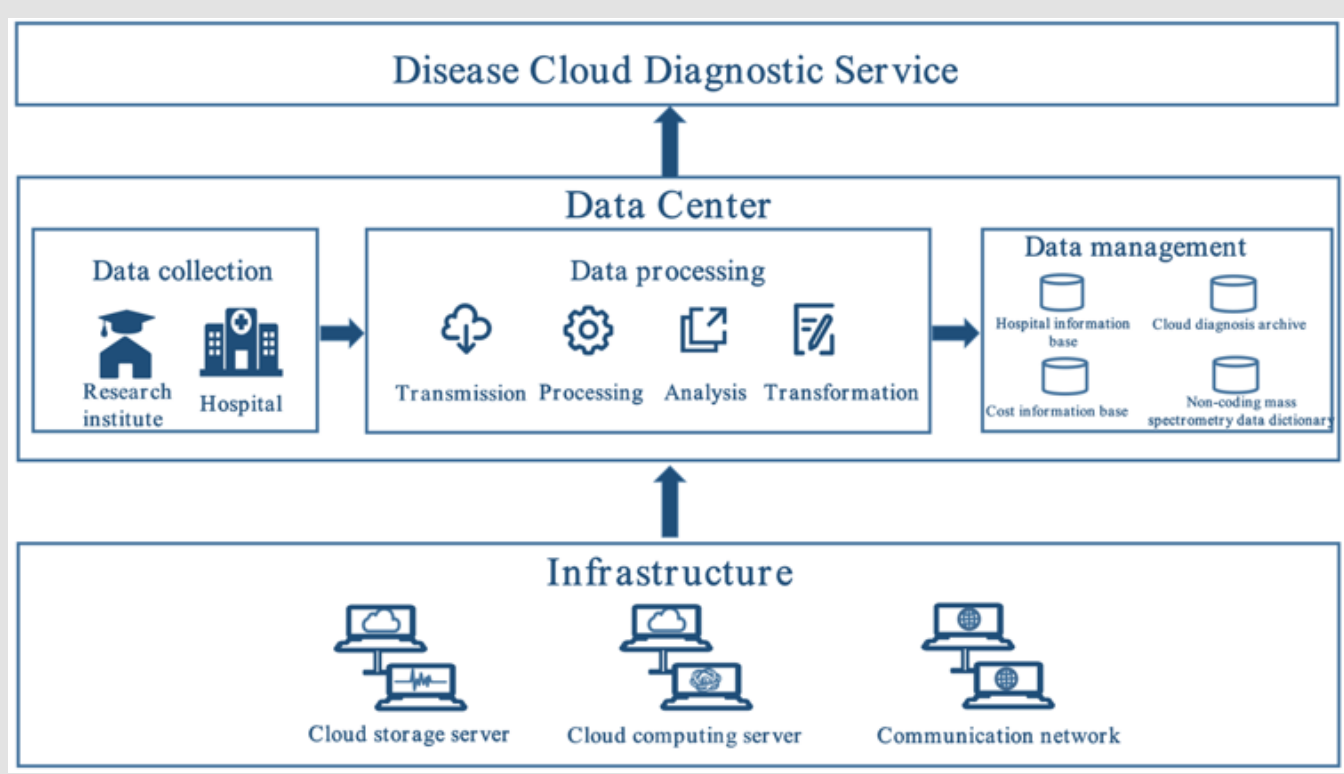

Figure 1: Functional Structure Diagram of Cloud Diagnostic Service Platform Information System.
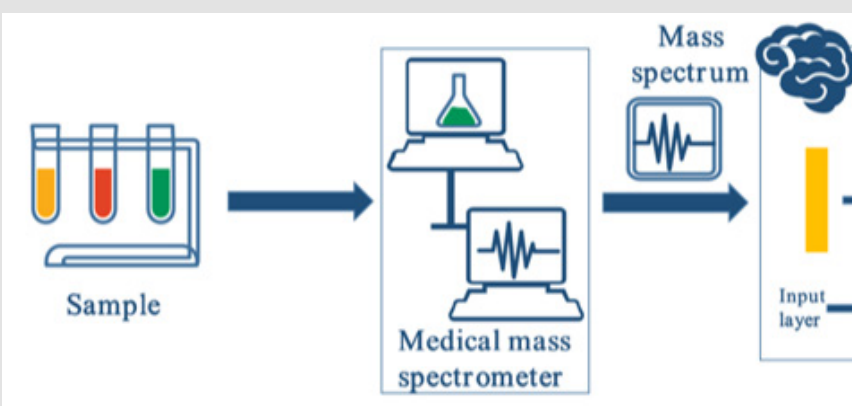

Deep convolutional neural network system
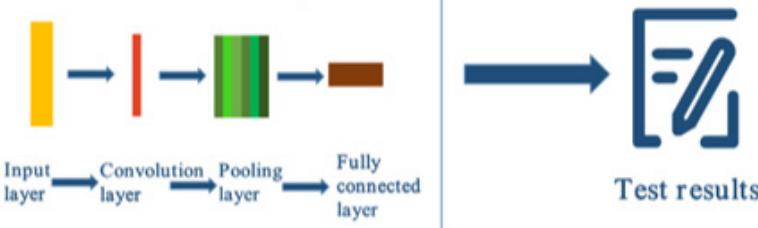

Test results

Figure 2: Mass Spectrometry - Deep Convolution Neural Network Identification Technique for Cancer Screening.

The detection samples are detected by mass spectrometer and output mass spectrum. The convolution neural network recognition system is used to analyze and input the cancer risk diagnosis results to achieve early cancer screening. By providing more mass spectrogram training for $\mathrm{CNN}$, the diagnosis equipment can learn and improve the results continuously, so as to achieve the effect of recognition and understanding of mass spectrogram by the diagnosis equipment. By reading enough mass spectrogram, the diagnosis equipment can learn to understand the image, have the ability to assist doctors to conduct more accurate analysis and judgment, and make this diagnosis cognition highly accurate in the future It has a very important value, which is conducive to improve the accuracy of cancer screening, and then improve the survival rate of cancer (Figure 2).

\section{Application of Shandong Non-coding Amino Acid Mass Spectrometry Diagnostic Platform}

For the cloud diagnosis service based on the non-coding amino acid mass spectrum data platform, the hospital applies to the cloud diagnosis service platform and provides the patient mass spectrum detection map, and the platform provides the diagnosis opinion and report through the big data cloud computing analysis. The operation process of cloud diagnosis service platform (Figure 2) is as follows:

A. Individuals apply to the hospital for medical grade mass spectrometry detection based on their own physical health and pay for detection and diagnosis fees.

B. The hospital accepts the individual detection application, and extracts the detection samples, and obtains the mass spectrum through the non-coding amino acid detection mass spectrometer for inspection and analysis. Then apply for cloud diagnosis service to cloud diagnosis service platform and pay for it, and then submit sample mass spectrum data.

C. The Cloud diagnosis service platform receives the application, processes the sample mass spectrum data through the computing center, obtains the diagnosis result, and issues the diagnosis result to the hospital. 


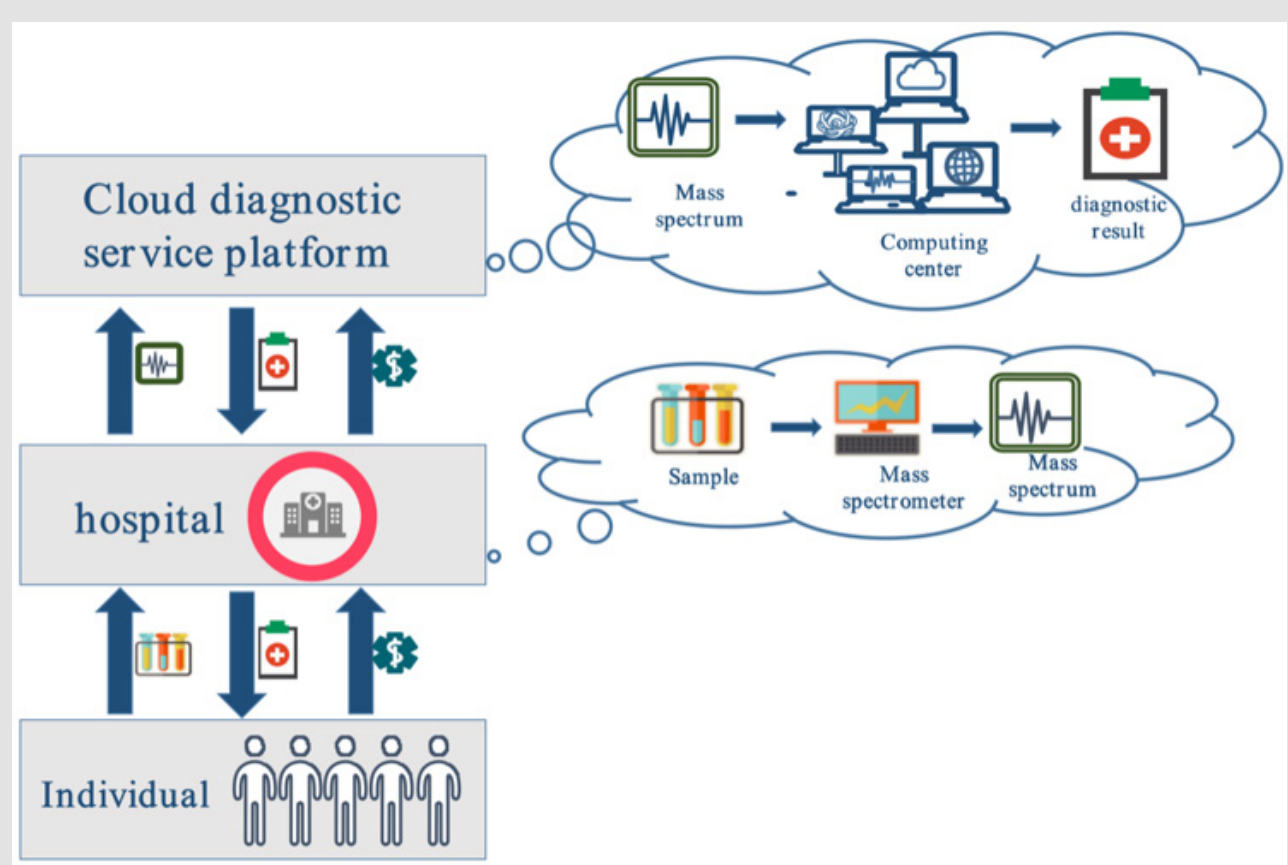

Figure 3: Operation Flow of Cloud Diagnostic Service Platform.

D. After receiving the diagnosis results, the hospital shall provide the patients with diagnosis opinions and reports in combination with the doctors' opinions (Figure 3).

The disease cloud diagnosis system uses modern communication, electronic and multimedia computer technology to realize the remote collection, transmission, processing, storage and query of non-coding amino acid mass spectrometry data, to help hospitals to implement accurate diagnosis. The system realizes the big data cloud diagnosis mechanism in precision medicine, reduces the difficulty of precision medicine in hospitals, and improves the accuracy and accuracy of disease diagnosis. The information system and operation mechanism of cloud diagnosis service platform based on non-coding amino acid mass spectrometry database provide development direction for big data health care and precision medicine.

\section{Acknowledgement}

This project is funded by Key Technology Research and Development Program of Shandong (2016CYJS1A01-3).

\section{References}

1. Terry SF (2015) Obama's precision medicine initiative. Genetic testing and molecular biomarkers 19(3): 113-114.
2. Robinson PN (2012) Deep phenotyping for precision medicine. Human mutation 33(5): 777-780.

3. Mirnezami R, Nicholson J, Darzi A (2012) Preparing for precision medicine. New England Journal of Medicine 366(6): 489-491.

4. Dai P, Wang Q Wang W, Jing R, Wang W, et al. (2016) Unraveling molecular differences of gastric cancer by label-free quantitative proteomics analysis. International journal of molecular sciences 17(1): E69.

5. Zhao Z, Azadzoi KM, Choi HP, Jing R, Lu X, et al. (2017) LC-MS/MS analysis unravels deep oxidation of manganese superoxide dismutase in kidney cancer. International journal of molecular sciences 18(2): E319.

6. LeCun Y, Bottou L, Bengio Y, Haffner P (1998) Gradient-based learning applied to document recognition. Proceedings of the IEEE p. 1-46.

7. Luo Y, Wang L, Wang J (2013) Developing proteomics-based biomarkers for colorectal neoplasms for clinical practice: Opportunities and challenges. PROTEOMICS-Clinical Applications 7(1-2): 30-41.

8. Szegedy C, Liu W, Jia Y, Sermanet P, Reed S, et al. (2015) Going deeper with convolutions. In Proceedings of the IEEE conference on computer vision and pattern recognition p. 1-9.

9. Suzuki K (2017) Overview of deep learning in medical imaging. Radiological physics and technology 10(3): 257-273. 


\section{ISSN: 2574-1241}

DOI: 10.26717/BJSTR.2020.24.004001

Xingyuan Wang. Biomed J Sci \& Tech Res

(C) Co This work is licensed under Creative

Submission Link: https://biomedres.us/submit-manuscript.php

$\begin{array}{ll}\text { BIOMEDICAL } & \text { Assets of Publishing with us } \\ \text { RESEARCHES } & \text { Global archiving of articles } \\ & \text { - Immediate, unrestricted online access } \\ & \text { - Rigorous Peer Review Process } \\ \end{array}$

